\title{
Rapid emergence of Mycobacterium tuberculosis bedaquiline resistance: lessons to avoid repeating past errors
}

\author{
To the Editor:
}

Bedaquiline (BDQ) has demonstrated potent clinical activity against multidrug-resistant (MDR) and extensively drug-resistant (XDR) Mycobacterium tuberculosis complex strains [1-3]. It has now been used in $>50$ countries, and it is estimated that $\sim 2500$ patients had been treated with BDQ by the end of 2015. In spite of its recent clinical use, there are few reports of BDQ-resistant strains $[4,5]$. Mutations in the rv0678 gene encoding the MmpL5 efflux pump repressor generate low-level BDQ resistance and clofazimine (CFZ) cross-resistance [6]. To our knowledge, this is the sole mechanism of BDQ resistance described in clinical strains $[4,5]$. Despite its introduction in France in 2011 for XDR-and MDR-tuberculosis (TB) treatment, we report herein four BDQ-resistant cases, and discuss strategies to avoid a surge of BDQ resistance.

In France, all MDR M. tuberculosis complex strains are sent to the National Reference Centre for Mycobacteria (NRC) for complete genotypic and phenotypic drug susceptibility testing (DST), including BDQ since 2014. BDQ minimum inhibitory concentration (MIC) was measured in 7H11 medium in polystyrene petri dishes for all strains that were resistant to a screening concentration of $64 \mathrm{mg} \cdot \mathrm{L}^{-1}$ in Lowenstein-Jensen medium. For each MIC measure, H37Rv was included as susceptible control. For all strains screened as BDQ resistant, the atpE and rv0678 genes were sequenced [4]. BDQ dry powder was supplied by Janssen (Issy-les-Moulineaux, France). The clinical history of patients harbouring BDQ-resistant strains was retrospectively abstracted from medical records. Ethics approval of the study protocol was granted by Bligny Hospital institutional review board (Briis-sous-Forges, France).

Between January 1, 2014 and December 31, 2015, 209 MDR M. tuberculosis complex strains (including 40 XDR-TB) were routinely tested at the NRC for BDQ susceptibility. Among these, four (2\%) had elevated BDQ MICs, $0.25-0.5 \mathrm{mg} \cdot \mathrm{L}^{-1}$, compared to $0.03 \mathrm{mg} \cdot \mathrm{L}^{-1}$ for the H37Rv reference strain. The genotypic and phenotypic characteristics of these four unrelated strains are summarised in table 1.

Patient 1, born in Pakistan, had no past history of TB or leprosy treatment and never received BDQ or CFZ. He was diagnosed with bone and pulmonary MDR-TB on arrival in France in January 2015. His strain was isolated before any TB treatment displayed BDQ resistance.

Patient 2 was treated for MDR-TB in Romania from September 2013 to November 2014, but has never received BDQ nor CFZ. He was diagnosed in France with cavitary lung MDR-TB in October 2015. The strain isolated at that time displayed BDQ resistance.

Patient 3 was treated in Georgia for 24 months from 2011 to 2013 for MDR-TB with ethionamide (ETH), para-aminosalicylic acid (PAS), capreomycin (CAP), cycloserine (CYC) and amoxicillin-clavulanate (AMC), a regimen subsequently supplemented with pyrazinamide (PZA) and BDQ for the last 4 months. In January 2014, he was diagnosed in France with cavitary lung XDR-TB, with a BDQ-resistant strain.

Patient 4 arrived in France from Georgia in 2013 after a 10-year history of TB treatment, with extensive bilateral cavitary lung lesions. In July 2013, he started treatment with PZA, amikacin (AMK), ETH, PAS, linezolid (LNZ), ethambutol (EMB) and BDQ. The strain isolated on arrival in France was only susceptible to AMK, PAS and LNZ, and had BDQ MIC in the normal range. In August 2013, EMB and LNZ were withdrawn, due to in vitro resistance and peripheral neuropathy, respectively, and imipenem (IMP)/AMC was introduced. He became sputum culture negative in October 2013. In December 2013, IMP/AMC was

@ERSpublications

Bedaquiline resistance appears rapidly both after treatment and in patients that have never been treated http://ow.ly/mxEM30604OF

Cite this article as: Veziris N, Bernard C, Guglielmetti L, et al. Rapid emergence of Mycobacterium tuberculosis bedaquiline resistance: lessons to avoid repeating past errors. Eur Respir J 2017; 49: 1601719 [https://doi.org/10.1183/13993003.01719-2016]. 
TABLE 1 Characteristics of the four bedaquiline-resistant Mycobacterium tuberculosis strains

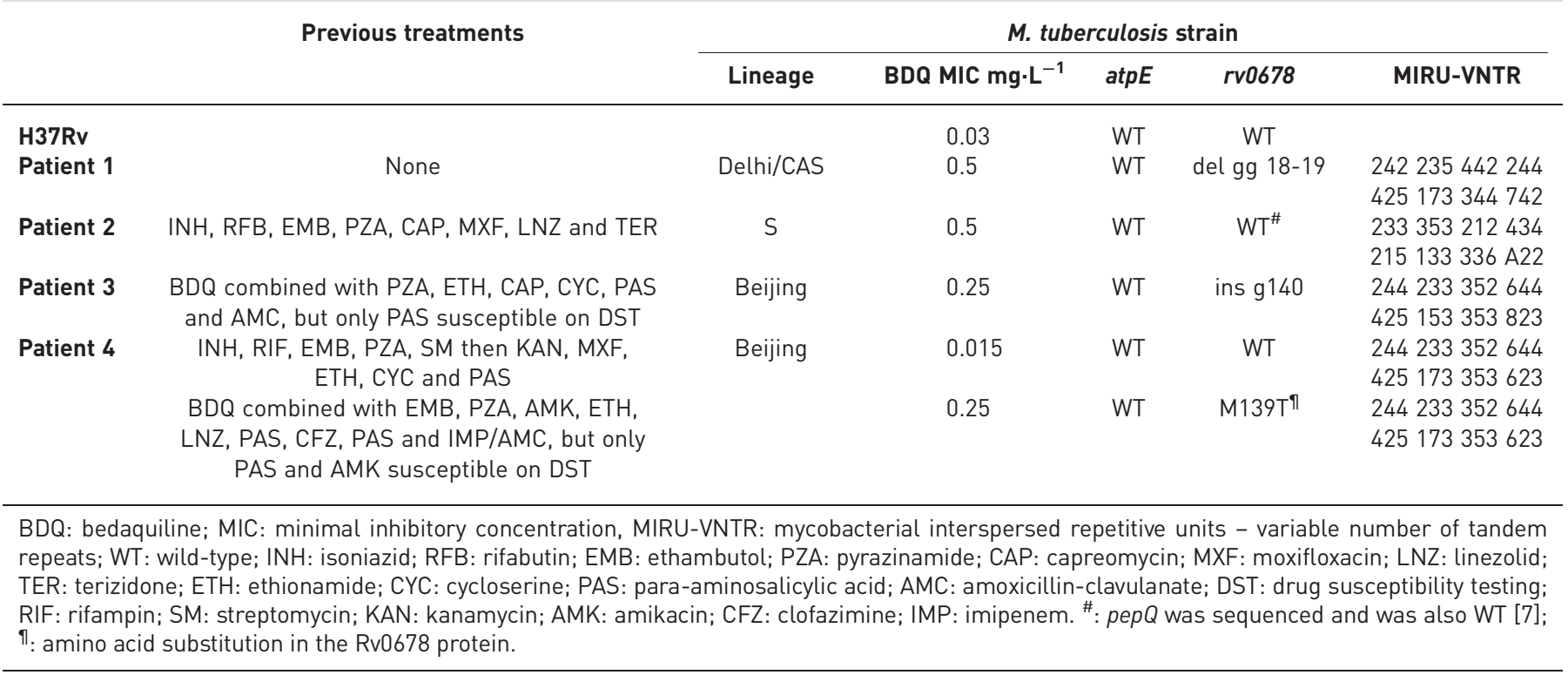

stopped and CFZ was introduced. In September 2014, AMK was stopped. In October 2014, the patient reverted to sputum culture positivity. DST of the 2014 strain showed the same susceptibility profile as the initial 2013 strain, except for the appearance of BDQ resistance.

The rapid appearance of BDQ resistance is alarming. These four cases can be grouped into two profiles, which raise different questions.

The first profile is that of patients 1 and 2, who had never been treated with BDQ. Considering that TB is transmitted from one patient to another, it can be hypothesised that BDQ resistance was selected by BDQ or CFZ treatment in the transmission chain. This is unlikely for BDQ, because of its recent introduction. However, CFZ may have been prescribed for a nontuberculous disease, such as leprosy. Finally, other compounds could select for BDQ resistance through $r v 0678$ mutations, because the recognised mechanism of resistance affects an efflux pump. Altogether, these data underline the importance of including BDQ DST in national programmes implementing BDQ for the treatment of MDR- or XDR-TB.

In patients 3 and 4 , the background regimen failed to prevent the selection of drug resistance. Reviewing DST results and treatment histories, it appears that except for BDQ, the only probable active drugs were PAS (for both patients) and AMK (for patient 4). We previously described a similar case in which fluoroquinolone resistance was selected, despite PAS and AMK co-administration [8]. Recently, a case of BDQ resistance was reported despite EMB, PAS, CAP, and CYC combined therapy [9]. Of note, when BDQ was switched for delamanid (DLM), DLM resistance was subsequently selected [9]. The 2013 World Health Organization recommendations on BDQ use do not address the effectiveness of BDQ companion drugs. Regimens in which $\mathrm{BDQ}$ is only combined with drugs with poor bactericidal activity or poor tissue diffusion, such as PZA, PAS, $\mathrm{CYC}, \mathrm{EMB}, \mathrm{CFZ}$ or aminoglycosides may not prevent the selection of BDQ resistance. We suggest that BDQ should be always associated with at least one drug with both bactericidal and sterilising activity for the full treatment duration in order to avoid selection of BDQ resistance. Fluoroquinolones, LNZ, DLM and possibly ETH may be considered in this category, since they have proven in vivo activity $[3,10]$. Furthermore, recent data suggest that the combination of DLM and BDQ is safe [11, 12]. If the number of remaining bactericidal drugs is low and there are important destructive lung lesions, surgery should be considered [13].

The definition of BDQ resistance is currently not completely established. The European Committee on Antimicrobial Susceptibility Testing adopted $0.25 \mathrm{mg} \cdot \mathrm{L}^{-1}$ as susceptibility breakpoint in 7H11 [14], which is above the MIC that inhibits $90 \%$ of the isolates or strains $\left(0.12 \mathrm{mg} \cdot \mathrm{L}^{-1}\right)$ [15]. However, with this breakpoint, the strain responsible for clinical failure in patient 4 (MIC $0.25 \mathrm{mg} \cdot \mathrm{L}^{-1}, 16$-fold higher than before BDQ treatment) would have been categorised as susceptible. The clinical impact of $r v 0678$ mutations conferring elevated MICs in the $0.25-0.5 \mathrm{mg} \cdot \mathrm{L}^{-1}$ range, i.e. below the usual BDQ serum level (3-4 mg. $\mathrm{L}^{-1}$ ) could be a matter of discussion. A murine experiment has shown that $r v 0678$ mutations increasing BDQ MICs by four- to eight-fold reduce the 1 -month BDQ bactericidal activity by $1 \log _{10}$ CFU [4]. Conversely, a clinical study suggested that there was no impact on treatment efficacy of a 
BDQ-containing combined regimen [3]. Nevertheless, the case of patient 4, who became initially sputum culture negative, but relapsed with positive cultures after 16 months of a BDQ-containing regimen, suggests that the clinical impact of such mutations could be meaningful. Finally, it seems that there is an overlap between MICs of wild-type and mutant strains, which may require a two-breakpoint classification with an intermediate category, as for other antituberculous drugs.

Nicolas Veziris $^{1,2}$, Christine Bernard ${ }^{1,2}$, Lorenzo Guglielmetti ${ }^{1,2,3}$, Damien Le Du ${ }^{3}$, Dhiba Marigot-Outtandy ${ }^{3,4}$, Marie Jaspard ${ }^{5}$, Eric Caumes ${ }^{5}$, Isabelle Lerat ${ }^{6}$, Christophe Rioux ${ }^{6}$, Yazdan Yazdanpanah ${ }^{6,7,8}$, Angelica Tiotiu', Nadine Lemaitre ${ }^{10}$, Florence Brossier ${ }^{1,2}$, Vincent Jarlier ${ }^{1,2}$, Jerome Robert ${ }^{1,2}$, Wladimir Sougakoff ${ }^{1,2}$ and Alexandra Aubry ${ }^{1,2}$ on behalf of the CNR MyRMA and the Tuberculosis Consilium of the CNR MyRMA ${ }^{11}$

${ }^{1}$ Sorbonne Universités, UPMC Univ Paris 06, Inserm, U1135, Centre d'Immunologie et des Maladies Infectieuses (CIMI-Paris), Paris, France. ${ }^{2}$ Centre National de Référence des Mycobactéries, Laboratoire de Bactériologie-Hygiène, Hôpitaux Universitaires Pitié-Salpêtrière-Charles Foix, APHP, Paris, France. ${ }^{3}$ Sanatorium, Centre Hospitalier de Bligny, Briis-sous-Forges, France. ${ }^{4}$ AP-HP, Hôpital Raymond Poincaré, Service de Médecine Aigue Spécialisée, Garches, France. ${ }^{5}$ AP-HP, Hôpitaux Universitaires Pitié-Salpêtrière-Charles Foix, Service des Maladies Infectieuses et Tropicales, Paris, France. ${ }^{6} \mathrm{AP}-\mathrm{HP}$, Hôpital Bichat, Services de Maladies Infectieuses et Tropicales, Paris, France. ${ }^{7}$ INSERM, IAME, UMR 1137, Paris, France. ${ }^{8}$ Université Paris Diderot, IAME, UMR 1137, Sorbonne Paris Cité, Paris, France. ${ }^{9}$ Dépt de pneumologie, CHU de Nancy, Vandouvre-lès-Nancy, France. ${ }^{10}$ Univ. Lille, CNRS, Inserm, CHU Lille, Institut Pasteur de Lille, U1019 - UMR, 8204 - CIIL - Center for Infection and Immunity of Lille, Lille, France. ${ }^{11}$ Full details of the members of the CNR MyRMA and Tuberculosis Consilium can be found in the Acknowledgements.

Correspondence: Nicolas Veziris, Centre National de Référence des Mycobactéries, APHP, laboratoire de BactériologieHygiène, 47-83 Bld de l'hôpital, 75013, Paris, France. E-mail: nicolas.veziris@aphp.fr

Received: July 262016 | Accepted after revision: Oct 292016

Support statement: The CNR MyRMA is supported by an annual grant from the Institut de Veille Sanitaire. N. Veziris, C. Bernard, L. Guglielmetti, F. Brossier, V. Jarlier, J. Robert, W. Sougakoff and A. Aubry work in a laboratory that has been financed by Janssen for preclinical work on bedaquiline. Janssen supplied bedaquiline dry powder.

Conflict of interest: Disclosures can be found alongside this article at erj.ersjournals.com

Acknowledgements: The members of CNR MyRMA (Centre National de Référence des Mycobactéries et de la Résistance des Mycobactéries aux Antituberculeux) are as follows: Emmanuelle Cambau, Laurent Raskine and Faïza Mougari (Assistance Publique Hôpitaux de Paris, Hôpitaux Universitaires Lariboisière-Saint Louis, Service de Bactériologie, Centre National de Référence des Mycobactéries et Résistance des Mycobactéries aux Antituberculeux (CNR-MyRMA), IAME, UMR 1137, INSERM, Univ Paris Diderot, Sorbonne Paris Cité, Paris, France).

The members of the Tuberculosis consilium of the CNR MyRMA are as follows: Claire Andrejak (Service de Pneumologie et de Réanimation Respiratoire, CHU Sud, Amiens, France), Mathilde Fréchet-Jachym (Sanatorium, Centre Hospitalier de Bligny, Briis-sous-Forges, France), Guillaume Thouvenin (Service de Pneumologie Pédiatrique, Hôpital Armand Trousseau, Assistance Publique Hôpitaux de Paris (AP-HP), Centre National de Référence des Maladies Respiratoires Rares RespiRare, Paris, France and Inserm UMRS928, Université Pierre et Marie Curie, Sorbonne Universités, Paris), Katarina Chadelat (Hôpital d'Enfants Armand-Trousseau, AP-HP, Paris), Bertrand Dautzenberg (Hôpitaux Universitaires Pitié-Salpêtrière-Charles-Foix, AP-HP, Université Pierre-et-Marie-Curie, Paris), Najoua Helali (Clinical Microbiology Unit, Saint-Joseph Hospital Network, Paris) and Bénedictine Rivoire (Samu Social, Paris).

\section{References}

1 Diacon AH, Pym A, Grobusch MP, et al. Multidrug-resistant tuberculosis and culture conversion with bedaquiline. $N$ Engl J Med 2014; 371: 723-732.

2 Guglielmetti L, Le Dû D, Jachym M, et al. Compassionate use of bedaquiline for the treatment of multidrug-resistant and extensively drug-resistant tuberculosis: interim analysis of a French cohort. Clin Infect Dis 2015; 60: 188-194.

3 Pym AS, Diacon AH, Tang S-J, et al. Bedaquiline in the treatment of multidrug- and extensively drug-resistant tuberculosis. Eur Respir J 2016; 47: 564-574.

4 Andries K, Villellas C, Coeck N, et al. Acquired resistance of Mycobacterium tuberculosis to bedaquiline. PLoS One 2014; 9: e102135.

5 Somoskovi A, Bruderer V, Hömke R, et al. A mutation associated with clofazimine and bedaquiline cross-resistance in MDR-TB following bedaquiline treatment. Eur Respir J 2015; 45: 554-557.

6 Hartkoorn RC, Uplekar S, Cole ST. Cross-resistance between clofazimine and bedaquiline through upregulation of MmpL5 in Mycobacterium tuberculosis. Antimicrob Agents Chemother 2014; 58: 2979-2981.

7 Almeida D, Ioerger T, Tyagi S, et al. Mutations in pepQ confer low-level resistance to bedaquiline and clofazimine in Mycobacterium tuberculosis. Antimicrob Agents Chemother 2016; 60: 4590-4599.

8 Veziris N, Martin C, Brossier F, et al. Treatment failure in a case of extensively drug-resistant tuberculosis associated with selection of a GyrB mutant causing fluoroquinolone resistance. Eur J Clin Microbiol Infect Dis 2007; 26: 423-425.

9 Bloemberg GV, Keller PM, Stucki D, et al. Acquired resistance to bedaquiline and delamanid in therapy for tuberculosis. N Engl J Med 2015; 373: 1986-1988.

10 Scardigli A, Caminero JA, Sotgiu G, et al. Efficacy and tolerability of ethionamide versus prothionamide: a systematic review. Eur Respir J 2016; 48: 946-952.

11 Lachâtre M, Rioux C, Le Dû D, et al. Bedaquiline plus delamanid for XDR tuberculosis. Lancet Infect Dis 2016; 16: 294. 
12 Tadolini M, Lingtsang RD, Tiberi S, et al. First case of extensively drug-resistant tuberculosis treated with both delamanid and bedaquiline. Eur Respir J 2016; 48: 935-938.

13 Mordant P, Henry B, Morel S, et al. Chirurgie et tuberculose multi/ultrarésistante: une revue de la littérature réhabilite une intervention adjuvante à l'antibiothérapie chez des patients sélectionnés [Adjuvant surgical resection for multidrug-resistant tuberculosis: a review]. Rev Mal Respir 2014; 31: 511-524.

14 European Committee on Antimicrobial Susceptibility Testing. Clinical Breakpoints. www.eucast.org/clinical_ breakpoints/ Date last accessed: April 5, 2016. Date last updated: January 2016.

15 US Food and Drug Administration. Anti-infective Drugs Advisory Committee Meeting Briefing Document. TMC207 (Bedaquiline): Treatment of Patients with MDR-TB. US Food and Drug Administration, 2012. 